\title{
THE ROLE OF ZERO SETS IN THE SPECTRA OF HYPONORMAL OPERATORS ${ }^{1}$
}

\author{
C. R. PUTNAM
}

Abstract. A compact set in the complex plane is the spectrum of a completely hyponormal operator if and only if the set has positive density.

1. Introduction. A bounded operator $T$ on a Hilbert space is said to be hyponormal if $T^{*} T-T T^{*} \geqq 0$, and completely hyponormal if there exists no nontrivial reducing space on which it is normal. It was shown in [5] that if $T$ is completely hyponormal and if its spectrum, $\operatorname{sp}(T)$, intersects an open disk in a nonempty set, then the (planar) measure of the intersection is positive. It will be shown below that, conversely, if $S$ is any compact set of the plane having positive density, in the sense that

$$
S \cap N \neq \varnothing \Rightarrow \operatorname{meas}_{2}(S \cap N)>0, \quad N \text { any open disk, }
$$

then there exists a completely hyponormal operator $T$ for which $S=\operatorname{sp}(T)$. Thus,

THEOREM 1. A necessary and sufficient condition that a compact set $S$ be the spectrum of a completely hyponormal operator is that (1.1) holds.

Consequently, sets of measure 0 play the same role for hyponormal operators as the nowhere dense compact sets $X$ for which $C(X)=R(X)$ play for subnormal operators; see [1].

As noted above, the necessity part of Theorem 1 was proved in [5], so that only the proof of the sufficiency remains. This will be given in $\$ 4$ as a consequence of Theorem 2 stated next, the proof of which will be given in $\$ 3$.

THEOREM 2. Let $S$ be a subset of the plane satisfying

$$
S \text { is compact and } \operatorname{meas}_{2}(S)>0 \text {. }
$$

Then there exists a completely hyponormal operator $M$ for which $M^{*} M-$ $M M^{*}$ has rank 1 and $\operatorname{sp}(M) \subset S$.

Received by the editors June 20, 1972 and, in revised form, March 26, 1973.

AMS (MOS) subject classifications (1970). Primary 47B20; Secondary 47A10.

Key words and phrases. Hyponormal operator, subnormal operator, spectrum.

${ }^{1}$ This work was supported by a National Science Foundation research grant 6460 50-1395515-31-74.

c) American Mathematical Society 1974 
A certain result, given below as a lemma and the validity of which remained problematic in [6], will be needed. (The author has since learned that the lemma follows from some results of R. O. Davies [4]; see \$2 below.) Some terminology will first be given. Let $\alpha$ denote any closed set of real numbers and let $S_{\alpha}$ denote the infinite closed strip $S_{\alpha}=\{z: \operatorname{Re}(z) \in \alpha\}$. For each $t, 0 \leqq t<2 \pi$, let $S(t, \alpha)=e^{i t} S_{\alpha}$, the set $S_{\alpha}$ rotated about the origin by the angle $t$.

Lemma. Let $S$ be a set of the plane for which (1.2) holds. Then there exists $a$ (finite or infinite) sequence $t_{1}, t_{2}, \cdots, 0 \leqq t_{n}<2 \pi$, and a sequence of compact sets $\alpha\left(t_{n}\right)$ on the real line for which the closed set

satisfies

$$
A=\bigcap_{n} S\left(t_{n}, \alpha\left(t_{n}\right)\right)
$$

$$
A \subset S \text { and } \operatorname{meas}_{2}(A)>0 .
$$

In case each $\alpha\left(t_{n}\right)$ is a closed interval, the set $A$ is convex (and closed). Thus the lemma states that any compact (hence, any measurable) set of positive planar measure contains a "generalized" compact convex subset of positive measure. It may be noted that the corresponding assertion concerning the existence of generalized rectangles is false; see [3].

2. Proof of the lemma. Choose an open rectangle $R$ with sides parallel to the coordinate axes and so that $S \subset R$. By choosing appropriate open vertical half-planes $\{z:-\infty<\operatorname{Re}(z)<a\}$ and $\{z: b<\operatorname{Re}(z)<\infty\}$ and a corresponding pair of horizontal half-planes one can "erase" the set $C-$ $R^{-}$. (Here $C$ denotes the complex plane and $R^{-}$denotes the closure of $R$.) Thus one may restrict attention to the set $R-S$, the complement of $S$ with respect to $R$. It is clearly sufficient to show that there exists a sequence of open intervals $\left(a_{n}, b_{n}\right)$, where $-\infty<a_{n}<b_{n}<\infty$, and real numbers $t_{n}$, where $0 \leqq t_{n}<2 \pi$, together with corresponding open strips

$$
S_{n}=e^{i t_{n}}\left\{z: a_{n}<\operatorname{Re}(z)<b_{n}\right\},
$$

for which

$$
(R-S) \subset\left(\bigcup_{n} S_{n}\right) \text { and } \operatorname{meas}_{2}\left(S-\left(\bigcup_{n} S_{n}\right)\right)>0 .
$$

The author is indebted to Professor R. O. Davies for informing him that the above desired result, hence the lemma, is essentially contained in Davies' paper [4]. In fact, it was shown there [loc. cit., p. 220] that if $E$ is any set of finite planar measure then there exists a planar measurable set $L$ composed of straight lines and satisfying $E \subset L$ and meas $_{2}(L-E)=0$. The proof of this last result depends on Lemma 6 of $[4$, p. 220]. A special 
case of that lemma will be needed in the present paper and will be formulated as the following:

Lemma (*) [R. O. DAviEs]. Let $R$ be an open rectangle $A B B^{\prime} A^{\prime}$ with horizontal sides $A B$ and $A^{\prime} B^{\prime}$, and let $E$ be any planar measurable set contained in $R$. Then, for any $\varepsilon>0$, there exists a sequence of open parallelograms $\left\{P_{n}\right\}, n=1,2, \cdots$, contained in $R$ and with two sides on $A B$ and $A^{\prime} B^{\prime}$ for which

$$
E \subset \bigcup_{n} P_{n} \text { and } \operatorname{meas}_{2}\left(\bigcup_{n} P_{n}-E\right)<\varepsilon
$$

First, it may be noted that, in Lemma 6 of [4], the corresponding sets $R$ and $P_{n}$ are all closed parallelograms. The formulation of Lemma (*) in which $R$ is an open rectangle and the $P_{n}$ are open parallelograms is a trivial modification and will be more convenient for our application below.

Next, let the rectangle $R$ and the set $E$ of Lemma (*) be identified with the sets $R$ and $R-S$ occurring in the beginning of this section. Further, for a given $\varepsilon>0$, let each parallelogram $P_{n}$ be extended to an open strip $S_{n}$ by simply removing its sides lying on $A B$ and $A^{\prime} B^{\prime}$ and extending the other two sides indefinitely in both directions. If $B=\bigcup_{n} S_{n}$, then, by (2.3),

$$
R-S \subset B \text { and } \operatorname{meas}_{2}(B \cap S)=\operatorname{meas}_{2}\left(\bigcup_{n} P_{n}-(R-S)\right)<\varepsilon \text {. }
$$

Since $\operatorname{meas}_{2}(S)>0$ and since $S=(S-B) \cup(S \cap B)$, relation (2.2) now follows by choosing $\varepsilon$ to be less than $\operatorname{meas}_{2}(S)$.

3. Proof of Theorem 2. Since $M$ can be replaced by $c M$ where $c=$ const $>0$, it can be supposed that $S$ is a subset of the closed unit disk. Let $T$ denote the unilateral shift operator, so that $\operatorname{sp}(T)$ is this disk and let $A$ satisfy (1.3) and (1.4) of the lemma. Next, note that $T$ and $A$ may be identified with the corresponding symbols in Theorem 2 of [6, p. 702]. Actually, it was assumed there that $\operatorname{sp}(T) \cap A$ had positive density, a condition not now assumed. Nevertheless, the proof given there [loc. cit., p. 706, bottom] shows that there exists an orthogonal projection $P$ for which $T_{P}=P T P$ is hyponormal; in fact, $T_{P}^{*} T_{P}-T_{P} T_{P}^{*}=P\left(T^{*} T-\right.$ $\left.T T^{*}\right) P$. Further, $\operatorname{sp}\left(T_{P}\right) \subset(\operatorname{sp}(T) \cap A)$ and $(\operatorname{sp}(T) \cap A)-\operatorname{sp}\left(T_{P}\right)$ is a set of measure 0 . (Here, $T_{P}$ as well as $T_{Q}$ below are regarded as operators on the corresponding projections of the original Hilbert space.) Since $\operatorname{sp}(T) \cap S=S$, it is clear from (1.4) that $\operatorname{sp}\left(T_{P}\right)$ differs from $A$ by a set of measure 0 . If $T_{P}$ is not already completely hyponormal one can choose (cf. [6, p. 707]) a projection $Q \leqq P$ so that $M=T_{Q}=Q T Q$ is completely hyponormal, $M^{*} M-M M^{*}$ has $\operatorname{rank} 1$, and $\operatorname{sp}(M)$ differs from $\operatorname{sp}\left(T_{P}\right)$, 
hence from $A$, by a set of measure 0 . But $\operatorname{sp}(M)$ must have positive density (necessity part of Theorem 1 ) and hence $\operatorname{sp}(M) \subset A(\subset S)$. This proves Theorem 2.

4. Proof of sufficiency in Theorem 1. This follows easily by taking direct sums of operators of the type $M$ in Theorem 2. Thus, since $S$ has positive density, there exists a sequence of points $\left\{z_{n}\right\}$ belonging to $S$ and which are dense in $S$, and open disks $N_{n}=\left\{z:\left|z-z_{n}\right|<r_{n}\right\}$ such that each set $S \cap N_{n}$ has positive measure. Then choose $M_{n}$ as in Theorem 2 so that $M_{n}$ is completely hyponormal, $M_{n}^{*} M_{n}-M_{n} M_{n}^{*}$ has rank 1 and $\operatorname{sp}\left(M_{n}\right) \subset\left(S \cap N_{N}^{-}\right)$. Then $T=\sum \oplus M_{n}$ is completely hyponormal and $\operatorname{sp}(T)$ is the closure of the set $\bigcup_{n} \operatorname{sp}\left(M_{n}\right)$; see [6, p. 703] for a similar argument. Since the $\left\{z_{n}\right\}$ are dense in $S$ it is clear that $\operatorname{sp}(T)=S$.

ADDED IN PROOF. It has recently been shown by R. W. Carey and J. D. Pincus (Construction of seminormal operators with prescribed mosaic (Preprint)), by other methods, that the operator occurring in the sufficiency part of Theorem 1 above can be chosen so as to have a onedimensional self-commutator.

\section{REFERENCES}

1. K. F. Clancey and C. R. Putnam, The local spectral behavior of completely subnormal operators, Trans. Amer. Math. Soc. 163 (1972), 239-244. MR 45 \#934.

2. - The spectra of hyponormal integral operators, Comm. Math. Helv. 46 (1971), 451-456.

3. R. Darst and C. Goffman, A Borel set which contains no rectangles, Amer. Math. Monthly 77 (1970), 728-729. MR 41 \#8615.

4. R. O. Davies, On accessibility of plane sets and differentiation of functions of two real variables, Proc. Cambridge Philos. Soc. 48 (1952), Part 2, 215-232. MR 13, 635.

5. C. R. Putnam, An inequality for the area of hyponormal spectra, Math. Z. 28 (1971), 473-477.

6. - The spectra of completely hyponormal operators, Amer. J. Math. 93 (1971), 699-708. MR 43 \#6757.

Department of Mathematics, Purdue University, Lafayette, Indiana 47907 